\title{
Complicações obstétricas e perinatais durante a pandemia do COVID-19
}

\author{
Obstetric and perinatal complications during the COVID-19 pandemic \\ Complicaciones obstétricas y perinatales durante la pandemia COVID-19
}

Recebido: 11/10/2021 | Revisado: 18/10/2021 | Aceito: 21/10/2021 | Publicado: 24/10/2021

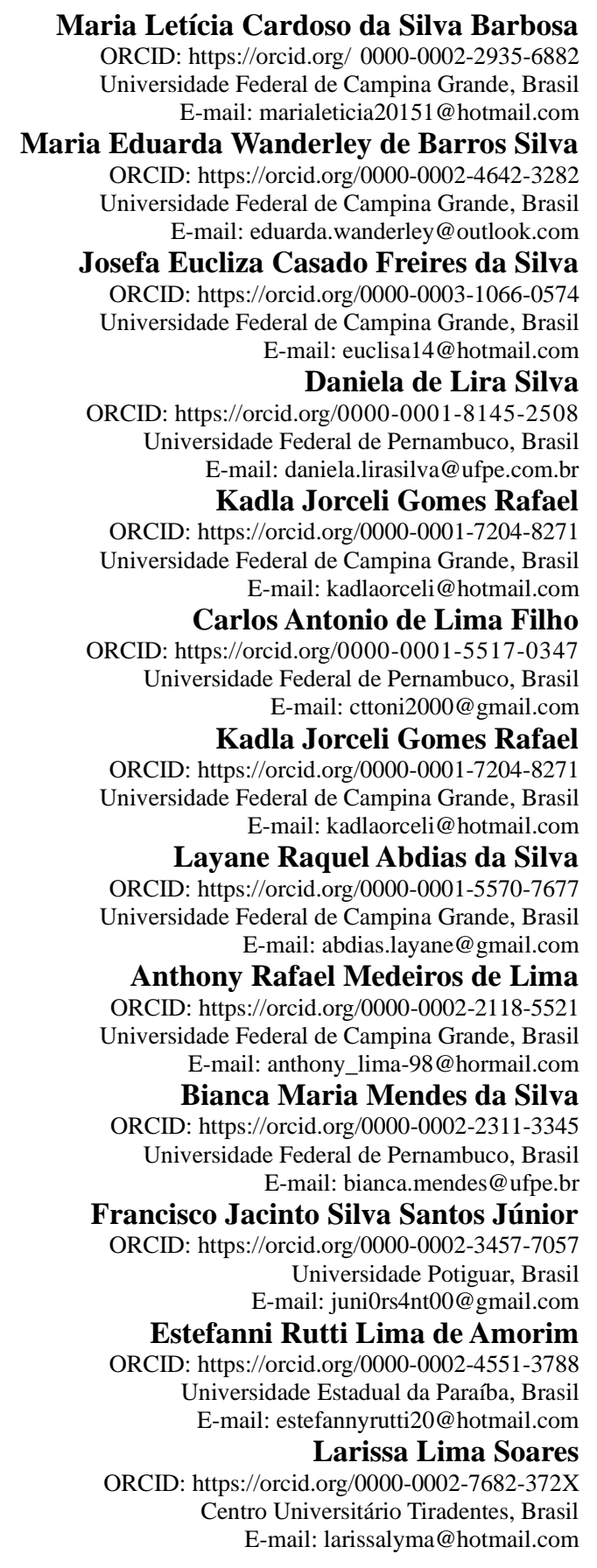

\section{Resumo}

O presente estudo trata-se de uma revisão literária que apresenta como objetivo identificar as complicações obstétricas e perinatais durante o período da pandemia do COVID-19 a fim de reduzir devidas consequências, onde desempenha um papel relevante na saúde integral do paciente proporcionando bem-estar e evitando efeitos adversos. A coleta de dados ocorreu entre os meses de agosto e setembro a partir da base de dados da Literatura Latino-Americana e do 
Caribe e Ciências da Saúde, Bases de Dados em Enfermagem e Medical Literature Analysis and Retrieval System Online. Para a estratégia de busca foram utilizados os descritores: pré-estabelecidos pelo Descritores em Ciências da Saúde (DECS), sendo "Complicações do Trabalho de Parto", "COVID-19”, "Infecção Puerperal" e "Complicações Infecciosas na Gravidez". Após o processo de elegibilidade dos estudos, foram identificados, analisados e utilizados 9 artigos publicados entre os anos de 2019 a 2021, referentes as complicações na gravidez e no período pós-parto ou puerperal. Deste modo, as informações coletadas na análise de estudo compõem diversas discussões atualizadas a respeito do tema, também elaboradas no cenário pandêmico pela COVID-19, que fomentou a pesquisa de revisão integrativa. Chega-se à conclusão que é importante ressaltar que devido as mudanças decorrentes da pandemia, o preparo profissional deve ser especial e deve-se oferecer as mulheres o suporte adequado e qualificado, dito isto, torna-se necessário que os profissionais conheçam os sintomas da COVID-19, para que auxiliem no manejo correto e apropriado no cuidado da gestante e do bebê. No entanto, vale ressaltar a importância de estudos acerca do tema, devido à escassez de estudos concretos, necessitando de pesquisas mais amplas e com uma necessidade para conhecer ainda mais os riscos que podem ser provocados durante o período gravídico-puerperal e perinatal.

Palavras-chave: Complicações do trabalho de parto; COVID-19; Infecção puerperal e complicações infecciosas na gravidez.

\begin{abstract}
This study is a literary review that aims to identify obstetric and perinatal complications during the period of the COVID-19 pandemic in order to reduce the consequences, where it plays a relevant role in the integral health of the patient providing well-being and avoiding adverse effects. Data collection took place between August and September from the Latin American and Caribbean Literature and Health Sciences, Databases in Nursing and Medical Literature Analysis and Retrieval System Online database. The following descriptors were used for the search strategy: preestablished by the Health Sciences Descriptors (DECS): "Labor Complications", "COVID-19", "Puerperal Infection" and "Infectious Complications in Pregnancy". After the eligibility process of the studies, we identified, analyzed and used 9 articles published between 2019 and 2021, referring to complications in pregnancy and in the postpartum or puerperal period. Thus, the information collected in the study analysis comprises several updated discussions on the theme, also elaborated in the pandemic scenario by COVID-19, which fostered the research of integrative review. It is concluded that it is important to emphasize that due to the changes resulting from the pandemic, professional preparation should be special and women should be offered adequate and qualified support, that being said, it is necessary that professionals know the symptoms of COVID-19 to assist in the proper and proper management of the care of the pregnant woman and the baby. However, it is worth mentioning the importance of studies on the subject, due to the scarcity of concrete studies, requiring more extensive research and with a need to know even more about the risks that can be caused during pregnancy-puerperal and perinatal.
\end{abstract}

Keywords: Labor complications; COVID-19; Puerperal infection and infectious pregnancy complications.

\title{
Resumen
}

El presente estudio es una revisión literaria cuyo objetivo es identificar las complicaciones obstétricas y perinatales durante el período de pandemia de COVID-19 con el fin de reducir las consecuencias, donde desempeña un papel relevante en la salud integral del paciente proporcionando bienestar y evitando efectos adversos. La recolección de datos tuvo lugar entre los meses de agosto y septiembre a partir de la base de datos de la Literatura Latinoamericana y del Caribe y Ciencias de la Salud, Bases de Datos en Enfermería y Medical Literature Analysis and Retrieval System Online. Para la estrategia de búsqueda fueron utilizados los descriptores pre-establecidos por el Descriptores en Ciencias de la Salud (DECS), siendo "Complicaciones del Trabajo de Parto", "COVID-19", "Infección Puerperal" y "Complicaciones Infecciosas en el Embarazo". Después del proceso de elegibilidad de los estudios, fueron identificados, analizados y utilizados 9 artículos publicados entre los años 2019 a 2021, referentes a las complicaciones en el embarazo y en el período posparto o puerperal. De este modo, las informaciones recogidas en el análisis de estudio componen diversas discusiones actualizadas acerca del tema, también elaboradas en el escenario pandémico por COVID-19, que fomentó la investigación de revisión integrativa. Se llega a la conclusión de que es importante resaltar que debido a los cambios derivados de la pandemia, la preparación profesional debe ser especial y se debe ofrecer a las mujeres el apoyo adecuado y calificado, dicho esto, se hace necesario que los profesionales conozcan los síntomas de la COVID-19, para que ayuden en el manejo correcto y apropiado en el cuidado de la gestante y del bebé. Sin embargo, vale resaltar la importancia de estudios acerca del tema, debido a la escasez de estudios concretos, necesitando de investigaciones más amplias y con una necesidad para conocer aún más los riesgos que pueden ser provocados durante el período gravídico-puerperal y perinatal.

Palabras clave: Complicaciones del trabajo de parto; COVID-19; Infección puerperal y complicaciones infecciosas del embarazo. 


\section{Introdução}

O ano de 2020 foi marcado pela pandemia do novo coronavírus, a mesma decretada pela Organização Mundial de Saúde (OMS), teve seu início na província de Hubei, na China que gerou a doença causada pelo vírus da Síndrome Respiratória Aguda Grave (SARS-CoV-2). Dessa forma, assim que foi decretado a pandemia do coronavírus a China, Espanha e Estados Unidos da América possuíam maior evidência no mundo visto que apresentavam um maior número de casos alertando assim os órgãos de saúde sobre a transmissibilidade do vírus (Souza et al., 2021).

Os diversos tipos de coronavírus como a Síndrome Respiratória Aguda Grave (SARS) e a Síndrome Respiratória do Oriente Médio (MERS) e atualmente a COVID-19 causam na humanidade desde sintomas gripais até doenças mais graves levando a complicações e até a morte de um grande contingente de pessoas. O coronavírus é conhecido por ser transmitido com rápida propagação em comparação com outros coronavírus sendo através de transmissão via gotículas respiratórias e pelo contato direto e indireto por fontes contaminadas causando dificuldade no controle do vírus (Acelas et al., 2021).

De acordo com a Organização Mundial de Saúde (OMS), as gestantes se apresentam como um grupo de risco para a COVID-19, pois, de acordo com a literatura, as gestantes que adquirem a infecção por SARS-CoV-2 podem evoluir para um quadro grave associado a uma comorbidade e, têm a possibilidade de passar por um parto cesariano de emergência ou um parto prematuro, elevando assim o risco de morte neonatal e materna (Estrela et al., 2020).

Além disso, o Ministério da Saúde (MS) incluiu todas as gestantes, puérperas, e pacientes que tenham tido perda gestacional ou fetal até 15 dias como um grupo de risco para a infecção causada pela COVID-19 (Costa et al., 2021).

Assim, as principais causas de morte materna no Brasil são causadas por hemorragia, hipertensão arterial, aborto e infecção puerperal, estas são consideradas como evitáveis. Estudos relatam algumas complicações que podem ocorrer com a mulher infectada pelo vírus, dentre elas, destacam-se o aumento da pré-eclâmpsia, alterações laboratoriais como a leucocitose, linfopenia, neutrocitose, anemia, aumento da procalcitonina, do D-dímero, da proteína C reativa e do Lactato Desidrogenase, o LDH (Souza et al., 2020).

Em um estudo realizado pelo Centro de Controle e Prevenção de Doenças dos Estados Unidos, as gestantes infectadas pela COVID-19 são mais vulneráveis a desenvolver a forma grave da doença, e tem mais chances de ter um parto prematuro ou padeceram uma perda gestacional. Esta pesquisa avaliou cerca de 1.872 gestantes infectadas, o que apontou uma ocorrência de $17 \%$ de prematuridade entre bebês de mães infectadas pelo vírus (Allotey et al., 2020). Isso acontece porque a gestação é vista como alterações fisiológicas, estas alterações quando relacionadas a COVID-19, aumenta de forma negativa o quadro de desfechos clínicos, pois, aumenta as incidências de pneumonias graves e síndrome respiratórias (Bhering et al., 2021).

O período puerperal é iniciado com o deslocamento da placenta ou quarto período de Greenberg, sendo prolongando até um ano após o parto, este fase envolve alterações imunológicas e hormonais, ainda mesmo que escassos os estudos referente ao puerpério de mulheres que foram diagnosticadas com COVID-19, algumas pesquisas chamaram a atenção das pesquisadoras, como o estudo de coorte realizado na Espanha que contou com a participação de 91 mulheres no ciclo gravídico-puerperal com diagnostico pela infecção do novo coronavírus, apenas quatro eram puérperas, sendo destas uma única mulher precisou ser hospitalizada (Barbero et al., 2020). Ainda, é descrito como um risco elevado para o tromboembolismo, mesmo que este seja um risco devido ao puerpério, quando relacionado com a COVID-19 a predisposição aumenta consideravelmente (Oliveira et al., 2020).

Como é novidade o SARS-CoV-2, existem poucas informações sobre sua disseminação, tratamento e controle com isso é desencadeado nas gestantes que estão dentro do contexto hospitalar, medo, insegurança e estresse sendo um desagradável estado mental produzido pela percepção de perigo iminente e desconhecido. As principais causas de estresse 
relacionadas ao vírus são a possibilidade de autoinoculação do vírus, preocupação com a possibilidade de infectar parentes e sensação de perigo (Fernández et al., 2021).

Portanto, existe a necessidade de que os profissionais sejam qualificados no atendimento ao parto, gestação e puerpério com retaguarda de emergências acessíveis funcionantes, ao colocar a maternidade segura como uma de suas prioridades com a finalidade de reduzir o insistente problema de mortalidade das mulheres durante o processo fisiológico da gravidez e do parto (Reganassi et al., 2015).

O presente estudo trata-se de uma revisão literária que apresenta como objetivo identificar as complicações obstétricas e perinatais durante o período da pandemia da COVID-19 a fim de reduzir devidas consequências, onde desempenha um papel relevante na saúde integral do paciente proporcionando bem-estar e evitando efeitos adversos.

\section{Metodologia}

Trata-se de uma revisão integrativa da literatura de abordagem qualitativa (Ludke \& André, 2013). Sendo um processo de análise, busca e descrição de um corpo de conhecimento em busca de uma resposta específica (Mendes, Silveira \& Galvão, 2008). Norteada pela seguinte questão de pesquisa "Quais as complicações obstétricas e puerperais durante a pandemia do COVID-19?". A coleta de dados ocorreu entre os meses de agosto e setembro a partir da base de dados da Literatura Latino-Americana e do Caribe e Ciências da Saúde (LILACS), Bases de Dados em Enfermagem (BDENF) e Medical Literature Analysis and Retrieval System Online (MEDLINE).

Para a estratégia de busca foram utilizados os descritores: pré-estabelecidos pelo Descritores em Ciências da Saúde (DECS), sendo "Complicações do Trabalho de Parto", "COVID-19", "Infecção Puerperal" e "Complicações Infecciosas na Gravidez", com isso sendo realizada as seguintes combinações: Complicações do Parto AND COVID-19 e Complicações Infecciosas na Gravidez AND Infecção Puerperal. Ressalta-se que as estratégias foram aplicadas conforme as características de cada base de dados. A amostra selecionada entre tantos disponível resultou em 56 artigos.

Os critérios de inclusão adotados foram: I) estudos clínicos e randomizados que respondessem à questão norteadora a partir da leitura do título e resumo; II) período de publicação entre os anos de 2019 a 2021; e III) estar nos idiomas português, inglês ou espanhol. Os critérios de exclusão envolveram estudos duplicados e que correspondessem a revisão integrativa, livros, cartas ao editor e artigos de nota prévia. Em seguida, foi selecionado o quantitativo de 9 artigos para compor o corpus de análise de artigos elegíveis.

A busca e seleção dos artigos foram realizadas e inicialmente identificou-se os estudos que apresentavam duplicidade entre as bases de dados pesquisadas e após isso, foi feita a avaliação do título e resumo. Por fim, foi realizada a leitura integral dos artigos identificados como potencialmente elegíveis, sendo incluídos no estudo, aqueles que estavam de acordo com os critérios de inclusão e exclusão.

\section{Resultados e Discussão}

A partir da elegibilidade dos estudos, respeitando os critérios de inclusão e exclusão, foram utilizados 9 artigos para a composição do corpus de análise, selecionando estudos os quais respondiam a pergunta norteadora e que foram publicados entre os anos de 2019 a 2021, considerando o período inicial da pandemia pela SARS-CoV-2 até Setembro de 2021. Os estudos mais atuais foram agrupados no Quadro 1, contendo informações sobre autoria e ano da publicação, título, delineamento metodológico e resultados. Delineando uma coleta de estudos atualizados, contribuindo para a pesquisa a identificação de complicações obstétricas e perinatais durante o período da pandemia do COVID-19, os artigos selecionados correspondem a revisões, estudos e séries de casos. 
Quadro 1. Corpus de análise de pesquisa. Brasil, 2021.

\begin{tabular}{|c|c|c|c|}
\hline $\begin{array}{c}\text { Autoria e ano de } \\
\text { publicação }\end{array}$ & Título do artigo & $\begin{array}{l}\text { Delineamento } \\
\text { metodológico }\end{array}$ & Resultados \\
\hline $\begin{array}{c}\text { CONTRERAS, N. et } \\
a l ., 2020 .\end{array}$ & $\begin{array}{c}\text { Induccíon de trabajo de } \\
\text { parto em pacientes COVID- } \\
\text { 19: experiencia em el } \\
\text { Hospital San Juan de Dios }\end{array}$ & Análise retrospectiva. & $\begin{array}{c}\text { Foi possível observar que as pacientes com PCR para } \\
\text { SARS-CoV-2 positivo, assintomáticos ou com doença } \\
\text { leve, foi possível realizar a indução do parto de acordo } \\
\text { com protocolos padronizados, alcançando taxas de } \\
\text { sucesso para partos vaginais semelhantes às de } \\
\text { pacientes sem a doença. }\end{array}$ \\
\hline $\begin{array}{l}\text { CZERESNIA, M.R. } \\
\text { et al.,2020. }\end{array}$ & $\begin{array}{c}\text { SARS-CoV-2 and } \\
\text { Pregnancy: A Review of the } \\
\text { Facts }\end{array}$ & Revisão de fatos. & $\begin{array}{l}\text { Experiências antecedentes apontam características } \\
\text { únicas na gravidez torna as mulheres grávidas mais } \\
\text { suscetíveis a complicações de infecções virais. No } \\
\text { entanto, isso não foi relatado com este novo vírus. } \\
\text { Porém, existem fatores de risco que parecem aumentar } \\
\text { a morbidade na gravidez, como obesidade (índice de } \\
\text { massa corporal [IMC]> 35), asma e doenças } \\
\text { cardiovasculares. Relatórios atuais descrevem um } \\
\text { aumento da taxa de parto prematuro e cesariana }\end{array}$ \\
\hline $\begin{array}{l}\text { LÓPEZ, P.A. et al., } \\
2020 .\end{array}$ & $\begin{array}{l}\text { Revisión de los riesgos } \\
\text { maternos y perinatales en } \\
\text { tiempos de COVID-19. } \\
\text { Desafíos para el rol de la } \\
\text { Matronería }\end{array}$ & $\begin{array}{l}\text { Revisão narrativa da } \\
\text { literatura. }\end{array}$ & $\begin{array}{l}\text { Nas mulheres grávidas, as formas graves de COVID-19 } \\
\text { acontecem na presença de doenças crônicas. No nível } \\
\text { perinatal, o maior risco é o parto prematuro, geralmente } \\
\text { por indicação médica e por cesariana. Até o momento } \\
\text { não há evidências de transmissão, mas, não se deve } \\
\text { descartar. Os riscos neonatais estão relacionados ao } \\
\text { contágio por proximidade e com medidas restritivas que } \\
\text { podem prejudicar o binômio mãe e filho. }\end{array}$ \\
\hline $\begin{array}{c}\text { SOUZA, A. S. R; } \\
\text { AMORIM, M, M, R, } \\
2021 .\end{array}$ & $\begin{array}{l}\text { Maternal mortality by } \\
\text { COVID-19 in Brazil }\end{array}$ & $\begin{array}{l}\text { Revisão da literatura } \\
\text { séries de casos. }\end{array}$ & $\begin{array}{c}\text { Diante disso, a preocupação com os cuidados às } \\
\text { gestantes e puérperas é imprescindível, principalmente } \\
\text { porque essa população apresenta dificuldades de acesso } \\
\text { ao pré-natal de qualidade, o que pirou ainda mais nesse } \\
\text { período de pandemia. }\end{array}$ \\
\hline $\begin{array}{c}\text { CORNEJO, N; } \\
\text { CANDIA.P, } 2020 .\end{array}$ & $\begin{array}{c}\text { Descripción de la aplicación } \\
\text { de screening universal para } \\
\text { SARS-CoV-2 en mujeres } \\
\text { embarazadas que ingresan } \\
\text { para interrupción en } \\
\text { Hospital de Carabineros - } \\
\text { Santiago de Chile }\end{array}$ & Análise descritiva. & $\begin{array}{l}\text { A realização do teste PCR para SARS-CoV-2 para } \\
\text { todas as mulheres grávidas que entram em um serviço } \\
\text { de Ginecologia e Obstetrícia ajuda a identificar } \\
\text { pacientes assintomáticas infectadas com o vírus. Como } \\
\text { o levantamento de sinais e sintomas não permite } \\
\text { identificar pacientes positivos, é necessário considerar a } \\
\text { realização de triagem universal nos protocolos de } \\
\text { admissão hospitalar em nosso país. }\end{array}$ \\
\hline $\begin{array}{l}\text { ROJAS. V. D, et } \\
\text { al.,2020. }\end{array}$ & $\begin{array}{l}\text { Pronóstico Perinatal em } \\
\text { embarazadas de tercer } \\
\text { trimestre recuperadas de } \\
\text { infección por COVID-19 }\end{array}$ & $\begin{array}{l}\text { Estudo retrospectivo } \\
\text { observacional. }\end{array}$ & $\begin{array}{l}\text { Os resultados sugerem que em casos selecionados onde } \\
\text { as características clínicas permitem, diferir a } \\
\text { interrupção da gravidez até após o período infeccioso } \\
\text { pode se associar a bons desfechos de morbimortalidade } \\
\text { materna e neonatal, entretanto, é fundamental continuar } \\
\text { as pesquisas. }\end{array}$ \\
\hline $\begin{array}{c}\text { VIELMA, O. S. et } \\
\text { al.,2020. }\end{array}$ & $\begin{array}{c}\text { Parto prematuro en } \\
\text { pacientes COVID-19 en } \\
\text { Hospital San Juan de Dios }\end{array}$ & $\begin{array}{l}\text { Estudo de coorte } \\
\text { observacional } \\
\text { retrospectivo. }\end{array}$ & $\begin{array}{l}\text { Entre as pacientes com COVID-19, foi observado um } \\
\text { aumento do risco de parto prematuro em comparação } \\
\text { com as pacientes sem a doença, com a elevação de risco } \\
\text { sendo significativa naquelas com sintomas e ainda mais } \\
\text { se eles tivessem doença grave. }\end{array}$ \\
\hline $\begin{array}{l}\text { HERNÁNDEZ, O. B. } \\
\text { et al.,2020. }\end{array}$ & $\begin{array}{l}\text { COVID-19 y embarazo no } \\
\text { Chile: Informe preliminar } \\
\text { del estudio multicéntrico } \\
\text { GESTACOVID }\end{array}$ & $\begin{array}{l}\text { Estudo de coorte } \\
\text { descritivo. }\end{array}$ & $\begin{array}{l}\text { Gestantes com COVID-19 e comorbidades como } \\
\text { diabetes, hipertensão crônica e obesidade mórbida } \\
\text { precisam de acompanhamento rigoroso e devem ser } \\
\text { objeto de pesquisas futuras. A transmissão vertical de } \\
\text { COVID-19 deve ser exaustivamente estudada para } \\
\text { definir os mecanismos e o tipo de infecção envolvida. }\end{array}$ \\
\hline $\begin{array}{l}\text { OROSTIZAGA, A.A. } \\
\text { et al, } 2020 .\end{array}$ & $\begin{array}{l}\text { Reporte de caso: Manejo } \\
\text { multidisciplinario en } \\
\text { gestante com embarazo de } \\
31 \text { semanas y COVID-19 } \\
\text { positivo com evolución } \\
\text { grave }\end{array}$ & Relato de caso. & $\begin{array}{l}\text { Em surtos epidêmicos anteriores, as gestantes } \\
\text { apresentavam elevado índice de letalidade e risco de } \\
\text { tratamento em terapia intensiva. Com base em uma } \\
\text { revisão de relato de caso, COVID-19 na gravidez está } \\
\text { associado a morbidade materna grave, especialmente } \\
\text { em mulheres com comorbidades associadas. }\end{array}$ \\
\hline
\end{tabular}

Fonte: Autores (2021). 
Um estudo, que avaliou os dados da planilha do Sistema de Informação de Vigilância Epidemiológica da Gripe (SIVEP Gripe) disponibilizado pelo Ministério da Saúde, mostrou que 978 gestantes e puérperas acusaram a SRAG por COVID-19 e dessas foi relatado uma taxa de letalidade de 12,7\% (Souza \& Amorim, 2021).

Em geral, os riscos maternos que estão associados ao SARS-CoV-2 estão relacionados a um perfil de alto risco devido a morbidades concomitantes à gravidez, como a asma, hipotensão e obesidade. Com isso, durante o processo de parto é necessário que sejam observados febre, sangramento e risco aumentado de ruptura prematura das membranas, nesse cenário a cesárea passa a ser altamente frequente podendo assim chegar a 80\% (López, Panjota, Mella, Utreras \& Vergara, 2020).

No entanto, além da associação com comorbidades e os fatores acima, o estudo revelou que foram identificadas falhas graves na assistência mostrando que 15\% das mulheres não tinham recebido qualquer tipo de assistência ventilatória, 28\% não tiveram acesso a leito de unidade e terapia intensiva (UTI) e 36\% não foram intubadas nem fizeram uso de ventilação mecânica (Souza \& Amorim, 2021). A relação da morte materna com a COVID-19 se constitui por várias variantes que envolvem o estado físico ao socioeconômico. No quadro 2 a seguir foram elencados alguns problemas que contribuem para a mortalidade materna no período pandêmico.

Quadro 2. Achados de fatores relacionados à mortalidade materna.

\begin{tabular}{c}
\hline $\begin{array}{c}\text { Fatores relacionados às complicações } \\
\text { maternas }\end{array}$ \\
Obesidade \\
Doenças cardiovasculares \\
Doenças respiratórias crônicas \\
Diabetes \\
Desigualdade racial, social e de gênero \\
Baixa qualidade de pré-natal \\
Violência obstétrica \\
Idade gestacional \\
\hline
\end{tabular}

Fonte: Autores (2021).

Ainda vale lembrar que, as complicações obstétricas estão correlacionadas com outras problemáticas, os fatores de riscos considerados pelo Ministério da Saúde como doenças crônicas do trato respiratório, cardiovasculares e obesidade propícia a forma mais grave e os índices de letalidade pela SARS-CoV-2. Porém, também se considera importante a questão da desigualdade social, racial e de gênero, que podem causar o aumento de mortes das grávidas e puérperas em condições de vulnerabilidade socioeconômica.

Em um estudo realizado em 2021, que visava analisar todos os óbitos publicados por COVID-19 no mundo, revelou que mulheres negras que foram hospitalizadas em condições de pior gravidade, como maior prevalência de dispneia e baixa saturação de oxigênio, além da elevada taxa de admissão na unidade de terapia intensiva (UTI) e de ventilação mecânica assistida, verificou ainda um risco de morte quase duas vezes maior em mulheres negras comparadas às brancas. A tamanha desigualdade social, racial e de gênero também foi descrita nos Estados Unidos da América (EUA), associada principalmente com o acesso aos serviços de saúde. Mostrando que as mortes maternas parecem de fato mais presentes nos países de baixa e média renda, sendo assim oriundo de falhas no sistema de saúde relacionadas aos determinantes sociais do processo saúdedoença (Souza \& Amorim, 2021).

Em relação ao caso clínico, existem diferenças significativas entre os sintomas de gestantes atendidas hospitalizadas e ambulatoriamente, pois os sintomas diferenciadores tem sido a dispneia, febre e tosse. Dessa forma, nesse estudo a dispneia 
esteve presente em $71 \%$ dos internados e apenas $10 \%$ dos pacientes ambulatoriais, já a febre com $29 \%$ dos pacientes, mas apenas em $20 \%$ dos pacientes ambulatoriais e $68 \%$ nos pacientes com manejo hospitalar. A tosse está presente em $78 \%$ das mulheres hospitalizadas, enquanto apenas em $25 \%$ das pacientes ambulatoriais. Isso faz com que coincida com a literatura que considera os casos clínicos de maior gravidade de acordo com os sintomas que os pacientes apresentam (Hernandez, Honorato, Silva \& Martínez, 2020).

Com diversos fatores relacionados às complicações obstétricas e o acometimento da morte materna, é possível identificar os danos, consequências presentes em grávidas e puérperas no processo de infecção pela COVID-19, mediante os estudos existentes. Considerando os resultados encontrados nos estudos os quais apresentam diferentes amostras que são bastante relevantes para qualificação da assistência perinatal durante a pandemia. Para se reduzir os riscos maternos e perinatais são recomendados a utilização dos atendimentos remotos a fim de reduzir o número de contatos face a face, no momento não foram encontrados estudos que relatavam essa prática e poucas experiências publicadas que buscam validar a modalidade remota tendo que o mesmo não substitui o método obstétrico e ginecológico, ou seja, o encontro pessoal, mas, se torna útil na redução de idas aos serviços de saúde.

Foi relatado que $86 \%$ das mulheres grávidas durante a pandemia apresentaram sintomas leves ou assintomáticos, 9,3\% sintomas moderados e 4,7\% sintomas graves. As considerações da doença leve foram definidas como nenhuma pneumonia ou pneumonia leve, a grave foi definida como frequência respiratória $>30$, SatO $2 \leq 93 \%$ e doenças críticas como choque séptico e insuficiência respiratória. Com isso houve a resolução da gravidez em 18 pacientes dos quais 56\% foram partos normais e dois pacientes tiveram pioras dos sintomas logo após o parto (Czeresnia, et al., 2020). No mesmo estudo, houve a notificação de 86 partos, sendo $92 \%$ cesáreos e $8 \%$ partos vaginais de acordo com os estudos a principal indicação da realização de cesáreas foi o sofrimento fetal embora os critérios utilizados para esse diagnóstico não foram tão claro na maioria dos estudos (Czeresnia, et al., 2020). É observado ainda mais o aumento do número de cirurgias cesarianas, o que pode levar a se atentar aos riscos de ocorrência de violência obstétrica, levando em conta que o cenário pandêmico apesar dos decretos e normas atualizadas, o binômio mãe-filho pode ser afetado a depender da condição da mãe perante a infecção e a equipe de saúde com as medidas restritivas do distanciamento.

Baseado nos relatos de casos em todo o mundo e revisões, a COVID-19 parece estar associado à morbidade materna grave, durante o período gestacional. Sendo um risco ainda maior em mulheres grávidas obesas e com comorbidades. Um caso mostrou uma grávida com infecção por SARS-COV2 no terceiro trimestre de gestação, com uma evolução grave que foi tratada de forma interdisciplinar, transferida para um centro mais especializado e complexo devido ao risco de deterioração respiratória materna e consequentemente a interrupção da gravidez, permitindo adequado suprimento respiratório e manejo materno-fetal (Orostizaga, Apablaaza, Garrido, Zúñiga, Vaccarezza \& Wittkop, 2020).

Em outro estudo, com 92 pacientes com COVID-19 no período infeccioso foi relatado que não houveram mortalidade materna com baixa admissão materna na UTI (4\%). Contudo, foi encontrado que houve um aumento de prematuridade, submissão a Unidade de Terapia Intensiva neonatal e parto cesáreo (Rojas, Manríquez \& Echeverria, 2020). Um dos fatores que indicam a prevalência da incidência na prematuridade é a gravidade da doença pois as pacientes com COVID-19 grave necessitaram de oxigênio, quanto aqueles com doenças críticas necessitaram de ventilação mecânica ou estavam em choque (Vielma, Lopez, Bustos, Assar \& Valdés, 2020).

Nesse contexto, é sugestivo questionamentos de todas as disciplinas, tais como as novas dificuldades que uma crise global de saúde pode acarretar. Para equipe multiprofissional de saúde, doulas e parteiras é de urgência o aprendizado qualificado deste novo patógeno e suas implicações na saúde materna, perinatal e neonatal. É de suma importância conhecer o novo coronavírus e como ele pode afetar o início ou evolução da gravidez, o processo de parto e nascimento, sendo assim podendo afetar o processo de amamentação, estado de saúde e a vida do recém-nascido, bem como o estado integral da mãe 
(Lopez, Pantoja, Mella, Utreras \& Vergara, 2020). No entanto, estudos comprovam que a amamentação deve ser estimulada, mesmo que a mãe esteja infectada pelo vírus, considerando que é um meio de passagem de anticorpos, pode assim, transmitir anticorpos ao bebê.

A preocupação com a atenção à gestante e à puérpera é imprescindível, tendo em vista que essa população apresenta dificuldades para acessar a qualidade do pré-natal, que se agravou mais ainda com o novo coronavírus. Não só o pré-natal, mais também toda a atenção à saúde da mulher está sendo prejudicada pela pandemia, seja pelo medo de algumas gestantes buscarem atendimento por incertezas e pelo medo de sair de casa, aumentando a frequência de sinais e sintomas de ansiedade e depressão, ou por erros graves significativos frequentes no atendimento à mulher nas cidades, priorizando o atendimento ao tratamento do COVID-19.

Analisando a situação no contexto hospitalar um estudo demonstrou a importância da realização do teste PCR para SARS-CoV-2 em todas as gestantes que ingressam no serviço de Ginecologia e Obstetrícia para interrupção, o que auxilia na identificação de pacientes assintomáticas infectadas com o vírus, por meio do levantamento de sinais e sintomas não é possível identificá-las em sua totalidade, ajudando assim a conduta da equipe de saúde e a organização da distribuição dos leitos hospitalares (Cornejo \& Candia, 2020).

Os desafios são referentes ao manejo da prevenção da infecção, na compreensão da consequência das medidas sanitárias, bem como isolamento e quarentena, direcionada a uma nova adaptação das mulheres grávidas. Levando em conta que necessita de apoio, qualidade de vida e conforto. No entanto, frente a hipermedicalização do trabalho de parto e puerpério, modifica o processo natural, o que prolonga a internação e pode deixar consideráveis impactos na saúde mental das mães no início e pós-parto. Estudos apontam um elevado aumento de depressão e ansiedade (Lopez, Pantoja, Mella, Utreras \& Vergara, 2020). Essa realidade ainda não pode ser medida, mas, espera-se grandes consequências no âmbito da saúde mental causada também pelo novo coronavírus e as medidas de isolamento (Souza \& Amorim, 2021).

\section{Conclusão}

As gestantes são caracterizadas como um grupo com suas particularidades, quando abordamos o contexto COVID-19, torna-se ainda mais vulnerável devido as modificações naturais que causa uma gestação. Objetivo da pesquisa foi alcançado, onde visava identificar as complicações obstétricas e perinatais durante o período da pandemia da COVID-19, sendo assim, é importante ressaltar que devido as mudanças decorrentes da pandemia, o preparo profissional deve ser especial e deve-se oferecer as mulheres o suporte adequado e qualificado, dito isto, torna-se necessário que os profissionais conheçam os sintomas da COVID-19, para que auxiliem no manejo correto e apropriado, ajudando numa futura prevenção do agravo da doença, bem como no cuidado da gestante e do bebê. No entanto, vale ressaltar a importância de estudos acerca do tema, devido à escassez de estudos concretos, necessitando de estudos mais amplos e com uma necessidade para conhecer ainda mais os riscos que podem ser provocados durante o período gravídico-puerperal e perinatal. Por isto, é extremamente conveniente uma equipe capacitada desde a atenção primária a gestante, até o rastreio dos casos e monitoramento da COVID-19.

\section{Referências}

Acelas, A. L. R., Getial, D. Y., \& Montañez-Cañon, W. (2021). Correlación entre diagnósticos, resultados e intervenciones de enfermeríaenel cuidado al paciente hospitalizado por COVID-19. Revista Cuidarte, 12:e1944. https://revistas.udes.edu.co/cuidarte/article/view/1944/2106

Allotey, J., Stallings, E., Bonet, M., Yap, M., \& Chatterjee, S. (2020). Clinical manifestations, risk factors, and maternal and perinatal outcomes of coronavírus disease 2019 in pregnancy: living systematic review and meta-analysis. BMJ, 370:m3320. https://www.ncbi.nlm.nih.gov/pmc/articl es/PMC7459193/pdf/bmj.m3320.pdf

Barbero, P., Muguerza, L., Herraiz, I., Burguillo, A. G., \& San Juan, R. (2020). SARS-COv-2 in pregnancy: characteristics and outcomes of hospitalized and non-hospitalized women due to COVID-19. J Matern Fetal Neonatal Med, 20:1-7. https://pubmed.ncbi.nlm.nih.gov/32689846/ 
Bhering, N. B. V., Arndt, C. G., Filho, D. A. P. G., Vita, D. T. P., \& Chagas, F. R. C. (2021). O parto prematuro induzido pela covid-19: uma revisão de literatura. Brazilian Journal of Health Review, 4 (2), 4401-4415. https://www.brazilianjournals.com/index.php/BJHR/article/viewFile/25638/20394

Contreras, N., Elgueta, R., López, M., \& Bravo, M. (2020). Inducción de trabajo de parto en pacientes COVID-19: experiencia en el Hospital San Juan de Dios. Revista Chil. Obstétrica Ginecológica, 85 (1). https://www.scielo.cl/scielo.php?script=sci_arttext\&pid=S0717-75262020000700006

Cornejo, N., \& Candia, P. (2020). Descripción de la aplicación de screening universal para SARS-CoV-2 en mujeres embarazadas que ingresan para interrupción en Hospital de Carabineros - Santiago de Chile. Revista Chil. Obstétrica Ginecológica, 85 (1):2-8. https://www.scielo.cl/scielo.php?script=sci_arttext\&pid=S0717-75262020000700002

Costa, T. P., Ferreira, E. S., Rodrigues, D. P., Neri, D. T., \& Soares, E. A. (2021). Os desafios da enfermagem obstétrica no início da pandemia da COVID-19 no Estado do Pará. Research, Society and Development, 10(3):e9510313042. https://rsdjournal.org/index.php/rsd/article/view/13042

Czeresnia, R. M., Trad, A. T. A., Britto, I. S. W., Negrini, R., Nomura, M. L., Pires, P., Costa, F. S da., Nomura, R. M. Y., \& Ruano, R. (2020). SARS-CoV-2 and Pregnancy: A Review of the Facts. Revista Brasileira de Ginecologia Obstet. 42 (09). https://www.scielo.br/j/rbgo/a/yXGWYQ8kS96vqqbHB8kVc9c/?lang=en\#

Estrela, F. M., Silva, K. K. A., Cruz, M. A., \& Gomes, N. P. (2020). Gestantes no contexto da pandemia da Covid-19: reflexões e desafios. Revista de Saúde Coletiva, 30(2):e300215. https://pesquisa.bvsalud.org/controlecancer/resource/pt/biblio-1125361?src=similardocs

Fernández, I. A. M., Moreno, S. C., Díaz, L. C., Gallegos-Torres, R. M., Fernández, J. A. M., \& Martínez, E. K. H. (2021). Fear, Stress, and Know led geregarding COVID-19 in Nursing Students and Recent Graduates in Mexico. Invest. Educ. Enferm, 39(5). https://revistas.udea.edu.co/index.php/iee/article/view/345514/20804846.

Hernández, O., Horonato, M., Silva, M. C., Sepúlveda-Martínez, A., Fuenzalida, J., \& Von Bischhoffshausen, S. (2020). COVID 19 y embarazo en Chile: Informe preliminar del estudio multicéntrico GESTACOVID. Revista Chil. Obstétrica Ginecológica, 85 (1). https://www.scielo.cl/scielo.php?script=sci_arttext\&pid=S0717-75262020000700011\&lang=pt

López, P., Pantoja, L., Mella, M., Utreras, M., \& Vergara, C. (2020). Revisión de los riesgos maternos y perinatales en tiempos de COVID-19. Desafíos para el rol de la Matronería. 85(1). https://www.scielo.cl/scielo.php?script=sci_arttext\&pid=S0717-75262020000700018

Ludke, M., \& André, M. E. D. A. (2013). Pesquisa em educação: abordagens qualitativas. (2.ed.), EPU.

Mendes, K. D. S., Silveira, R. C. C. P., \& Galvão, C. M. (2008). Revisão integrativa: método de pesquisa para a incorporação de evidências na saúde e na enfermagem. Texto e contexto - Enfermagem, 17(4), 758-764. https://doi.org/10.1590/S014-07072008000400018.

Oliveira, K. F de., Oliveira, J. F de., Wernet, M., Paschoini, M. C., \& Ruiz, M. T. (2021). Período pós-parto e infecção pelo novo coronavírus: revisão de escopo. Revista Enfermagem UERJ, 29:e56037. https://docs.bvsalud.org/biblioref/2021/05/1151921/periodo-pos-parto-pt.pdf

Orostizaga, A., Apablaza, F., Garrido, B., Zúñiga, P., Vaccarezza, I., \& Wittkopf, D. (2020). Reporte de caso: Manejo multidisciplinario en gestante con embarazo de 31 semanas y COVID-19 positivo con evolución grave. Revista Chil. Obstétrica Ginecológica, 85(1). https://www.scielo.cl/scielo.php?script=sci_arttext\&pid=S0717-75262020000700013

Reganassi, C., Barros, K. C. S., Katch, M., \& Nogueira, L. D. P. (2015). Mortalidade materna: desafios para enfermagem no enfrentamento da assistência. Revista Fafibe On-line, 8(1), 319-331. https://unifafibe.com.br/revistasonline/arquivos/revistafafibeonline/sumario/36/30102015190327.pdf

Rojas, D. V., Carreño Manríquez, L., \& Díaz Echeverría, C. (2020). Pronóstico Perinatal en embarazadas de tercer trimestre recuperadas de infección por COVID-19. Revista Chil. Obstétrica Ginecológica, 85 (1). https://pesquisa.bvsalud.org/portal/resource/pt/biblio-1138645

Souza, A. S. R., \& Amorim, M. M. R. (2021). Maternal mortality by COVID-19 in Brazil. Revista Brasileira de Saúde Materno Infantil. 21 (1) 253-256. https://www.scielo.br/j/rbsmi/a/R7MkrnCgdmyMpBcL7x77QZd/?lang=en\#

Souza, H. C. C., Matos, M. M. R de., Costa, R. A., Lima, M. A. C., Cardoso, A. S., \& Bezerra, M. M. (2020). COVID-19 e gestação: manifestações clínicas, alterações laboratoriais e desfechos maternos, uma revisão sistemática de literatura. Brazilian Journal of Health Review, 3(6). https://www.brazilianjournals.com/index.php/BJHR/article/view/19623

Souza, S. S., Cunha, A. C., Suplici, S. E. R., Zamprogna, K. M., \& Laurindo, D. L. P. (2021). Influência da cobertura da atenção básica no enfrentamento da covid-19. Journal Health NPEPS, 6(1), 1-21. https://periodicos.unemat.br/index.php/jhnpeps/article/view/4994

Vielma, S., López, M., Bustos, J. C., Assar, R., \& Valdés, F. (2020). Parto prematuro en pacientes COVID-19 en Hospital San Juan de Dios. Revista Chil. Obstétrica Ginecológica, 85 (1):59-66. https://www.scielo.cl/scielo.php?script=sci_arttext\&pid=S0717-75262020000700009 